\title{
Immirzi Ambiguity, Boosts and Conformal Frames for Black Holes
}

\author{
Luis J Garay and Guillermo A Mena Marugán \\ Centro de Física Miguel A Catalán, IMAFF, CSIC, \\ Serrano 121, 28006 Madrid, Spain
}

\begin{abstract}
We analyze changes of the Immirzi parameter in loop quantum gravity and compare their consequences with those of Lorentz boosts and constant conformal transformations in black hole physics. We show that the effective value deduced for the Planck length in local measurements of vacuum black holes by an asymptotic observer may depend on its conformal or Lorentz frame. This introduces an apparent ambiguity in the expression of the black hole entropy which is analogous to that produced by the Immirzi parameter. For quantities involving a notion of energy, the similarity between the implications of the Immirzi ambiguity and a conformal scaling disappears, but the parallelism with boosts is maintained.
\end{abstract}

PACS numbers:04.70.Dy, 04.60.Ds, 03.30.+p

Almost thirty years ago, a combination of classical and semiclassical arguments led to the conclusion that black holes possess thermodynamic properties [1]-[4]. In particular, a study of the mass change produced by infinitesimal variations of the black hole parameters revealed a close similarity with the first law of thermodynamics if one identifies the entropy $S$ and temperature $T$ with the area $A$ and surface gravity $\kappa$ of the black hole horizon [2]. Based partly on these results, Bekenstein proposed to regard $A$ as really providing a gravitational entropy [3]. At that time, however, no radiative process associated with a black hole was known from which one could infer a physical notion of temperature. Besides, the formula for the mass variation [2] allowed to assign the formal roles of temperature and entropy only up to an ambiguity in a constant [1]. In this context, Hawking performed a semiclassical analysis using methods of quantum field theory in curved backgrounds [4]. He proved that the presence of a black hole results in a thermal radiation with $T=\kappa \hbar /(2 \pi c)$. Here, $\hbar$ and $c$ are the Planck constant and the speed of light (and the Boltzmann constant has been absorbed in the definition of $T$ ). Hence, a black hole has an entropy determined by the Bekenstein-Hawking $(\mathrm{BH})$ formula $S=A /\left(4 l_{P}^{2}\right)$, where $l_{P}=\sqrt{\hbar G / c^{3}}$ is the Planck length and $G$ is the Newton constant.

A statistical explanation for this entropy has been only recently suggested in terms of the number of quantum states corresponding to a macroscopic black hole configuration $[5,6]$. Among the explanations proposed, we will concentrate our attention on that inspired in loop quantum gravity [6]. In this case, the states counted are spin networks compatible with the horizon geometry. It is generally admitted that this derivation of the entropy formula is affected by an ambiguity in a positive factor. In fact, loop gravity is based on the Ashtekar formulation of general relativity [7] in which the phase space is described by a real $S U(2)$ connection and a densitized triad. This real connection can be obtained as the difference between the spin connection of the triad and the extrinsic curvature (in triadic form) [7]. Nonetheless, a completely equivalent classical description is reached if, in the above construction, one multiplies the extrinsic curvature by a positive 
constant $\beta$ and divides the densitized triad by the same factor. This factor is usually called the Immirzi parameter [8].

Regardless of the value of $\beta$, one can quantize the theory representing quantum states as spin networks [9]. In this framework, one can promote the area of a surface to a positive operator that has a discrete spectrum and is well defined at least at the kinematical level $[10,11]$. For black holes, the boundary conditions ensure that the operator for the horizon area is also dynamically consistent, providing an observable [6, 12]. The remarkable fact realized by Immirzi is that quantization procedures based on connections that differ in the value of $\beta$ lead to different area spectra [8]. Actually, the Immirzi parameter appears as a global factor in the eigenvalues of the horizon area, and it is only the spectrum of $A_{\beta} / \beta$ that is independent of the selected quantization. We have introduced the notation $A_{\beta}$ to indicate that the area is calculated in the formulation with Immirzi parameter equal to $\beta$. The counting of quantum states then leads to the black hole entropy $S=\beta_{0} A_{\beta} /\left(4 \beta l_{\star}^{2}\right)[6]$, where $\beta_{0}=\ln 2 /(\pi \sqrt{3})$ and the length $l_{\star}$ is determined by the fact that the Hilbert-Einstein (HE) term in the gravitational action is divided by $16 \pi l_{\star}^{2} /(c \hbar)$. Given this definition, $l_{\star}$ is commonly identified with $l_{P}$. In any case, it is clear that one must demand $\beta l_{\star}^{2}=\beta_{0} l_{P}^{2}$ to recover the $\mathrm{BH}$ formula.

The roots and significance of the Immirzi ambiguity are not fully understood [13]. As far as the kinematics of general relativity is concerned, it is equivalent to the ambiguity in a constant scale transformation, which can be viewed as a change of conformal frame with constant conformal factor [14]. Nevertheless, this equivalence is broken when the Hamiltonian dynamics is taken into account. In addition, the Immirzi ambiguity has been argued to arise from the partial gauge fixing performed in Ashtekar gravity in terms of real connections, where a time gauge is chosen, reducing the gauge group from $S O(3,1)$ to $S U(2)$ [15]. One of the reasons for the confusion about the origin of this ambiguity is that, while the black hole entropy depends on the Immirzi parameter in loop quantum gravity, no ambiguity seems to arise in the standard deduction of the $\mathrm{BH}$ formula [16]. Our aim in this work is to show that, contrary to this belief, there exist in fact certain ambiguities which, rather than the value of the entropy, affect the empirical definition of the Planck length. As a result, this length becomes in practice an effective scale whose value (in terms of $l_{\star}$ ) may vary with the frame employed. We will consider frames that are related by constant conformal transformations and inertial frames in the asymptotic flat region of the black hole which are not at rest. We will analyze the role of these conformal transformations and boosts in black hole physics and compare them with a change of the Immirzi parameter in Ashtekar gravity.

Let us first study the effect of conformal transformations. We consider two frames with line elements related by $d \tilde{s}^{2}=\Omega^{2} d s^{2}$, where the conformal factor $\Omega>0$ is constant. Obviously, this factor can be absorbed by a dilatation of the unit of length, because the line element has dimension of area. Hence, we can think of $d s^{2}$ and $d \tilde{s}^{2}$ as representing the same spacetime but in systems of coordinates that simply differ in their length scale. One would thus expect that the predictions and measurements of two observers, each regarding his respective conformal frame as the physical one, were related just as dimensional analysis suggests. Let us show that this expectation holds for black holes.

It is trivial but lengthy to repeat the semiclassical derivation of the $\mathrm{BH}$ formula in the new conformal frame $d \tilde{s}^{2}$. Nonetheless, we can easily understand the main consequences of this change. Suppose $d s^{2}$ is the line element of a black hole spacetime with unit timelike 
Killing field $\xi^{\mu}$ with respect to which we define the black hole mass $M$. In the new frame, the unit Killing field is $\tilde{\xi}^{\mu}=\xi^{\mu} / \Omega$ and, as a consequence of this new normalization, the mass transforms as $\widetilde{M}=M / \Omega$, i.e., exactly as a scaling of the unit of length would dictate (keeping $\hbar$ and $c$ fixed). The Hawking temperature $T$, on the other hand, can be obtained, up to a constant factor, as the inverse of the period in imaginary time of the scalar Green functions for the black hole background [16]. A conformal transformation of coordinates, and in particular of time, makes this period scale with $\Omega$. Therefore, $\widetilde{T}=T / \Omega$. Note that the black hole mass and temperature transform in the same way, as expected since they both have dimensions of energy. The differential mass formula $d M=T d S$ (here restricted to the neutral non-rotating case for simplicity) and its counterpart for the new conformal frame then imply that the black hole entropy is invariant, $\widetilde{S}=S$, confirming what one would anticipate on dimensional grounds.

Obviously, the horizon area scales as the line element under a constant conformal transformation, $\widetilde{A}=\Omega^{2} A$. Taking into account the invariance of the black hole entropy $\widetilde{S}=S=A /\left(4 l_{P}^{2}\right)$, we see that the effective value deduced for the Planck length in the new conformal frame is $\widetilde{l}_{P}=\Omega l_{P}$, just what one could expect from a dilatation. Assuming that $\hbar$ and $c$ are fundamental constants, in the sense that they are frame-independent, we can view this transformation as a change in the effective dimensionful Newton constant. Actually, suppose that we define the gravitational action adopting a certain conformal frame as a universal frame of reference, e.g. $d s^{2}$. The coefficient of the HE term is $D \equiv \hbar c /\left(16 \pi l_{\star}^{2}\right)$, and the value of the Newton constant is $G=c^{4} /(16 \pi D)=l_{\star}^{2} c^{3} / \hbar$ in this frame. Under a constant scaling $d s^{2}=d \tilde{s}^{2} / \Omega^{2}$, the HE term gets divided by $\Omega^{2}$. The effective dimensionful Newton constant in the new conformal system is then $\widetilde{G}=\Omega^{2} G$, and hence $\widetilde{l}_{P}=\Omega l_{P}$.

This result may seem striking because it is frequently argued that the value of the Planck length is experimentally unambiguous (although there is an open debate about the significance of dimensionful constants in physics [17]). Let us analyze the determination of $l_{P}$ to clarify this issue. Accepting that $c$ and $\hbar$ are fundamental constants, we can introduce a system of units by only adopting a time unit (e.g. the second sec), because its product by $c$, and $\hbar / c^{2}$ times its inverse, provide length and mass units. We may define the time unit as a multiple of the period of an atomic transition (e.g. between two hyperfine levels of the ground state of ${ }^{133} \mathrm{Cs}$ ). Then, we can measure the experimental value of Newton constant, which in our system of units will be given by a dimensionless number multiplied by $(s e c)^{2} c^{5} / \hbar$. If we change to another conformal frame, as we have discussed above, periods will get scaled by the conformal factor $\Omega$ and, as a consequence, the time unit defined by the new observer will differ from the original one by this factor $(\widetilde{s e c}=\Omega s e c)$. The measured dimensionful Newton constant (not its dimensionless value) will then be scaled by $\Omega^{2}$, and the deduced Planck length will indeed be $\widetilde{l}_{P}=\Omega l_{P}$.

In conclusion, although a change of conformal frame that amounts to a constant scaling leaves invariant the value of the black hole entropy, it introduces a certain degree of ambiguity in the expression of this entropy as a function of the horizon area, namely $S=\tilde{A} /\left(4 \Omega^{2} l_{P}^{2}\right)$, where $l_{P}$ is the Planck length in the chosen frame of reference $d s^{2}$ and $\tilde{A}$ is the horizon area measured in the frame $d \tilde{s}^{2}=\Omega^{2} d s^{2}$.

We now discuss the effect of boosts, for which we consider two observers $O$ and $O^{\prime}$ in the asymptotic flat region of a black hole spacetime. Both observers employ systems 
of asymptotically Cartesian coordinates where they are at rest, $(t, \vec{x})$ and $\left(t^{\prime}, \vec{x}^{\prime}\right)$, with $\partial_{t}$ being a Killing field. The observer $O^{\prime}$ moves with constant velocity $\vec{V}$ with respect to $O$, so that the systems are related by a boost with parameter $\gamma=1 / \sqrt{1-(V / c)^{2}}[18]$, where $V=|\vec{V}|$ is the Cartesian norm of $\vec{V}$. In general, this Lorentz transformation leaves the spacetime metric invariant only in the asymptotic flat region. To study the consequences of Lorentz invariance, we will thus restrict our analysis to that region. Furthermore, we will disregard measurements that require an infinite amount of time for the transmission of the collected information to the observer (through the asymptotic flat region), because they are not feasible [19]. In practice, this means that we will only consider local measurements. This rationale eliminates, for instance, observations of the black hole effects made from different angles in the asymptotic region, because such measurements would imply infinite spatial separations.

Local measurements in the asymptotic flat region that indicate the presence of a vacuum black hole are those that detect the outward flux of Hawking radiation. The two observers $O$ and $O^{\prime}$ will see a thermal flux of particles, but their records will differ in the apparent temperature. The difference is due to the Doppler redshift that frequencies experiment in the boosted frame with respect to $O$ [16], i.e., with respect to the observer whose time coordinate corresponds to a Killing field. It is known that frequencies are shifted by a boost according to the law $\nu^{\prime}=\alpha \nu[18]$, where

$$
\alpha=\gamma\left(1-c^{-1} V \cos \varphi\right)
$$

and $\varphi$ is the angle formed by $\vec{V}$ and the direction of propagation in the rest frame of the source. In our case, Hawking radiation propagates radially and its rest frame corresponds to that of $O$. Note that, from our comments above, $\varphi$ is a constant in the region where the observer $O^{\prime}$ performs her (local) measurements. On the other hand, if we let $\vec{V}$ vary by analyzing different boosted observers, $\alpha$ may take any positive real value.

Owing to this redshift, the radiation detected by $O^{\prime}$ possesses the apparent temperature $T^{\prime}=\alpha T$. The same redshift affects also the energy density observed in the presence of the Hawking flux, $\rho^{\prime}=\alpha \rho$. To show this fact, we consider the stress-tensor in the Unruh vacuum that describes the Hawking evaporation process, $\left\langle T_{\mu \nu}\right\rangle$ [16]. Since $\partial_{t}$ is a Killing field, $\left\langle T_{\mu t}\right\rangle$ is a conserved quantity. The energy density seen by an observer with unit four-velocity $u^{\mu}$ is then $u^{\mu}\left\langle T_{\mu t}\right\rangle / c^{2}$ (we express $t$ in units of time). For simplicity, we will analyze only black holes with spherical symmetry. In this case, the only nonvanishing components of $\left\langle T_{\mu t}\right\rangle$ in the asymptotic region (adopting spherical coordinates for $O)$ are $\left\langle T_{t t}\right\rangle=-c\left\langle T_{r t}\right\rangle=\pi c T^{2} /\left(12 \hbar l_{P}^{2}\right)$ [16]. The energy density for $O$ is determined by $\rho=\left\langle T_{t t}\right\rangle / c^{2}$. On the other hand, since the four-velocity of $O^{\prime}$ is $(\gamma, \gamma \vec{V})$ [in $(t, \vec{x})$ coordinates], the density detected by the boosted observer is precisely $\rho^{\prime}=\alpha \rho$.

Suppose now that, given the fundamental constants $\hbar$ and $c$, the observer $O^{\prime}$ used the relation $l_{P}^{2}=\pi T^{2} /(12 \rho \hbar c)$ to determine the Planck length, but taking as experimental values of the energy density and the temperature its records $\rho^{\prime}$ and $T^{\prime}$. She will arrive at the apparent Planck length $l_{P}^{\prime}=\sqrt{\alpha} l_{P}$. On the other hand, if the black hole entropy has a statistical mechanical origin, its value must be given by the logarithm of the number of allowed quantum states. This number of degrees of freedom must not depend on the Lorentz frame considered, in the same way as we have shown that it is independent of the conformal frame. Then, $O$ and $O^{\prime}$ must agree in the value of the entropy, $S^{\prime}=S$. From 
this perspective, by using the $\mathrm{BH}$ formula the boosted observer would assign the effective area $A^{\prime}=\alpha A$ to the black hole horizon.

It is worth remarking that $A^{\prime}$ is only an effective, operationally defined area, that differs from $A$ as a result of the redshift caused by the boost. Actually, the true area of the black hole horizon in the boosted frame continues to be $A$, but this area is not directly observable from the asymptotic flat region. To see that the geometric area of the horizon does not change, let us analyze the most general stationary vacuum black hole, namely the Kerr solution, expressed in Boyer-Lindquist coordinates $(t, r, \theta, \phi)[1]$, where $t$ coincides with the time employed in the asymptotic region by $O$. The horizon has the topology of $S^{2} \times \mathbb{R}$. The sphere $S^{2}$ admits the coordinates $\theta$ and $\psi=\phi-\Omega_{H} t \in S^{1}$, while $\mathbb{R}$ is a null direction that can be described by the coordinate $t$. In this coordinate system, the degenerate metric on the horizon is diagonal and $t$-independent. Here, $\Omega_{H}$ is the constant coordinate angular velocity on the horizon [1]. The areas that we want to compare are those of the horizon sections of constant $t$ and $t^{\prime}$ (for $O$ and $O^{\prime}$ ). In principle, one might think of a possible discrepancy between these areas because, on the sections of constant $t^{\prime}$, the coordinate $t$ might vary modifying the area element. However, this does not occur, since $t$ corresponds to a null direction. Hence, the geometric area remains indeed the same.

Summarizing, a boosted observer in the asymptotic flat region that could carry out only local measurements on the Hawking radiation would infer an effective value of the Planck length that is either contracted or dilated. The value of the black hole entropy, nonetheless, should be invariant. In addition, we emphasize that the boost does not affect the geometric area of the horizon, in contradistinction to the Fitzgerald-Lorentz contraction experimented by the area of surfaces in flat spacetime [18].

Finally, we consider changes of the Immirzi parameter in Ashtekar gravity. In terms of the spacetime metric, the change from the unit parameter to $\beta$ can be realized as the transformation from the element $d s^{2}$ (with induced three-metric $h_{i j}$, lapse $N$, and shift vector $N^{i}$ ) to [14]

$$
d \bar{s}^{2}=-\beta^{-2} N^{2} d t^{2}+h_{i j}\left(d x^{i}+N^{i} d t\right)\left(d x^{j}+N^{j} d t\right)
$$

Since the induced metric is not modified, the transformation preserves the area of all constant- $t$ surfaces in the classical theory and, in particular, the geometric area $A$ of a black hole horizon. The scaling of the lapse has nevertheless an important consequence, namely, it changes the normalization of any (asymptotic) timelike Killing field. As a result, energies are affected by a redshift according to the Tolman relation $\bar{E}=\sqrt{\left(g_{t t} / \bar{g}_{t t}\right)} E$, where $g_{\mu \nu}$ denotes the spacetime metric. In the case of Hawking radiation, the shifted temperature and energy density are then $\bar{T}=\beta T$ and $\bar{\rho}=\beta \rho$. Here, we have taken as reference values those of the original frame with unit Immirzi parameter. We therefore find a complete parallelism with the situation described for boosts if we identify the parameters $\alpha$ and $\beta$. Note, in addition, that both parameters are positive but otherwise arbitrary.

In loop quantum gravity, as we have commented, the black hole entropy can be calculated counting quantum states, obtaining $S=\beta_{0} A_{\beta} /\left(4 \beta l_{\star}^{2}\right)$. Although the horizon area $A_{\beta}$ is classically independent of the Immirzi parameter, its discrete quantum spectrum is proportional to this constant. From this viewpoint, the area eigenvalues transform under 
a change of $\beta$ exactly as the value of the effective area $A^{\prime}$ under a change in the parameter $\alpha$ associated with Lorentz boosts. Furthermore, from the expression of the black hole entropy and the $\mathrm{BH}$ formula, each Immirzi description can be regarded as leading to a different effective value $l_{P}(\beta)=l_{\star} \sqrt{\beta / \beta_{0}}$ for the Planck length [14]. Introducing the definition $l_{P} \equiv l_{\star} / \sqrt{\beta_{0}}$ as a fixed reference value, we then get the transformation rule $l_{P}(\beta)=\sqrt{\beta} l_{P}$. In this way, the scaling laws for our effective quantities reproduce those deduced for boosts with the identification of parameters $\alpha$ and $\beta$.

This parallelism suggests the possibility that distinct Immirzi formulations correspond in fact to descriptions associated with different boosted frames. In this sense note that, as remarked in Ref. [20], a Lorentz boost connects different canonical theories, just like a change of Immirzi description. According to our conjecture, each Immirzi representation in loop quantum gravity would provide an area spectrum measured in terms of a different effective Planck length. The definition of this effective length incorporates the gravitational redshift introduced by the Immirzi parameter. This redshift is the same that would affect the local measurements of a boosted observer in a locally flat region where Lorentz invariance can be regained. In this sense, the quantity that should be Lorentz invariant is not the apparent value of the area (for any surface determined in a dynamically consistent way), but rather the corresponding number of elementary "geometric excitations", which is a dimensionless variable. This is exactly what happens with the Immirzi ambiguity, since the area spectrum differs only in the apparent value of the dimensionful quantum of area. It is precisely because of this property that the black hole entropy, when expressed as a dimensionless quantity, becomes independent of the selected Immirzi description.

Composing the above transformation laws, we can check that the introduction of the Immirzi parameter $\beta$ followed by a conformal scaling with factor $\Omega=\beta^{-1 / 2}$ indeed leaves the kinematics of Ashtekar gravity invariant [14]. The horizon area (spectrum) and the Planck length, which are quantities that can be defined in a kinematical context [14], remain unaltered by the composed transformation. The Hawking temperature and energy density are however modified, since they involve a concept of energy. This result and the analogy between boosts and changes of Immirzi description imply also that there must exist a composition of a boost and a conformal dilatation that preserves the value of the Planck scale, in agreement with recent works [21].

In conclusion, we have argued that the value of the Planck length determined by local measurements in a vacuum black hole spacetime depends on the conformal and Lorentz frame of the asymptotic observer. The value of the black hole entropy, on the other hand, must be frame-independent, because it only accounts for the number of quantum degrees of freedom compatible with the macroscopic configuration. Via the $\mathrm{BH}$ relation, the effective ambiguity in the Planck length then translates into an apparent ambiguity in the dimensionful magnitude of the horizon area. This ambiguity is similar to that caused by the Immirzi parameter in loop quantum gravity. We have seen that the kinematical effects produced in black hole physics by a boost, a constant conformal scaling, and a change of the Immirzi parameter are in fact analogous. For the case of the scaling, the analogy is broken as soon as dynamical considerations (such as measurements of energy) are brought into the scene. Remarkably, a complete parallelism is however encountered between the consequences of Lorentz boosts and transformations between Immirzi formulations. Based on this result, we have suggested the possibility that the various Immirzi representations of loop quantum gravity provide physical descriptions in different boosted frames, each of 
them associated with a different effective Planck length that takes into account the redshift introduced. We believe that this conjecture and its implications for Lorentz invariance and quantum gravity deserve further examination which will be carried out elsewhere.

Finally, note that our arguments have been restricted to vacuum black hole spacetimes, which possess an asymptotic flat region where invariance under boosts is attained. Such an asymptotic region needs not exist in more general spacetimes with a Killing horizon, e.g. when the spatial hypersurfaces are compact. In this latter case, however, the definitions of energies and temperatures are already affected by an ambiguity in a multiplicative constant at an early stage of the discussion, because there is no natural way to fix the normalization of any timelike Killing vector in the absence of the asymptotic region. Actually, this freedom in the normalization translates into the possibility of scaling the null normal to the horizon by a constant, a scaling that can be interpreted as the result of a boost, but now performed at the horizon.

The authors acknowledge DGESIC for financial support under Research Project No. BFM2001-0213.

\section{References}

[1] Wald R M 1984 General Relativity (Chicago: University of Chicago Press)

[2] Bardeen J M, Carter B and Hawking S W 1973 Commun. Math. Phys. 31161

[3] Bekenstein J D 1973 Phys Rev. D 72333

Bekenstein J D 1974 Phys Rev. D 93292

[4] Hawking S W 1975 Commun. Math. Phys. 43199

[5] Strominger A and Vafa C 1996 Phys. Lett. B 37999

Maldacena J and Strominger A 1996 Phys. Rev. Lett. 77428

[6] Ashtekar A, Baez J, Corichi A and Krasnov K 1998 Phys. Rev. Lett. 80904

Ashtekar A, Baez J C and Krasnov K 2001 Adv. Theor. Math. Phys. 41

[7] Ashtekar A 1991 Lectures on Non-Perturbative Canonical Gravity ed L Z Fang and R Ruffini (Singapore: World Scientific)

Thiemann T 2001 Introduction to Modern Canonical Quantum General Relativity Preprint gr-qc/0110034

[8] Immirzi G 1997 Nucl. Phys. Proc. Suppl. 5765

Immirzi G 1997 Class. Quantum Grav. 14 L177

[9] Rovelli C and Smolin L 1995 Phys. Rev. D 525743

Rovelli C 1998 Living Rev. Rel. 11

[10] Rovelli C and Smolin L 1995 Nucl. Phys. B 442593

Rovelli C and Smolin L 1995 Nucl. Phys. B 456753

[11] Ashtekar A and Lewandowski J 1997 Class. Quantum Grav. 14 A55 
[12] Ashtekar A, Corichi A and Krasnov K 2000 Adv. Theor. Math. Phys. 3419

[13] Rovelli C and Thiemann T 1998 Phys. Rev. D 571009

Gambini R, Obregón O and Pullin J 1999 Phys. Rev. D 59047505

Samuel J 2001 Phys. Rev. D 64048501 (2001)

Mena Marugán G A 2002 Class. Quantum Grav. 19 L63

[14] Garay L J and Mena Marugán G A 2002 Phys. Rev. D 66024021

[15] Alexandrov S 2000 Class. Quantum Grav. 174255

Alexandrov S 2002 Phys. Rev. D 65024011

[16] Birrell N D and Davies P C W 1986 Quantum Fields in Curved Space ed P V Landshoff et al. (Cambridge: Cambridge University Press)

[17] Duff M J, Okun L B and Veneziano G 2002 JHEP 0203023

[18] French A P 1968 Special Relativity (New York: Norton)

[19] To be more precise, we disregard measurements that require an unbounded amount of time when the asymptotic flat region is approached

[20] Amelino-Camelia G 2002 On the Fate of Lorentz Symmetry in Loop Quantum Gravity and Noncommutative Spacetimes Preprint gr-qc/0205125

[21] Magueijo J and Smolin L 2002 Phys. Rev. Lett. 88190403 